\title{
Opinion
}

\section{Some Stray Thoughts on doing Science under the Lockdown and the State of Quantum Theory of Solids}

\author{
SUSHANTA DATTAGUPTA \\ INSA Senior Scientist at the Bose Institute, Kolkata
}

The present unusual time of the Covid-19 pandemic has made many of us scientists homebound, restless and despondent. I, on the other hand - and I hope I am not amongst a minority - try to find the best out of a 'bad' situation. Staying in isolation with 'social distancing' maintained - there are fewer phone calls, and no meetings and committee-work. In addition, because of the stoppage of flights, air-travel - a part and parcel regimen of our scientists - is now nonexistent. For a retired scientist like me, these lifestylechanges have already occurred, of course, and I begin to think that the new situation is a 'blessing in disguise'.

At the outset, I must emphasize that I am a theoretical physicist - so I do not feel handicapped by my inability to go to the lab. I started to think that in all these years of so-called 'active life', I had spent an enormous amount of time in solving "others' problems" - had to take decisions in committees which were unpalatable to some, thereby creating 'enemies', because nothing of committee deliberations in our country remain confidential. I am now a free man, I finally have time - time to think, which was at a premium earlier.

The lockdown existence meant that I could choose my research topics and collaborations, on the internet, of course. Unbridled thinking also allowed me over the last few months to make forays into certain domains of physics, for which I did not seem to have the 'time' earlier. Besides, the pressure of seeing through $\mathrm{Ph}$. D. students within UGC-CSIRstipulated timeframe and tailor-making topics for them to make these tractable, inevitably meant that I could not concentrate earlier on things that I really wanted to do. One of these is Quantum Mechanics and its marvellous manifestations in properties of solids. Where does this subject stand today in our country is something I pondered on, in these lockdown times, which I want to share with you. My thoughts were triggered by the recent loss of two individuals, discussed below, who left an indelible mark on this topic.

It is interesting to note that unlike Classical Physics and even Atomic and Nuclear Physics, Solid State Physics (SSP) is not that old as an established subject. As Quantum Mechanics made its triumphant entry in the last century through the first "Thirty years of physics that shook the world" (George Gamow 1966), a few stalwarts quickly took the topic to noteworthy applications. These were however sporadic attempts of which mention must be made of John C. Slater on writing the wave function for multielectron systems as determinants (1929), Felix Bloch's exploitation of the periodicity of crystals in arriving at a momentum space description of electrons (1929), Linus Pauling's path-breaking contribution to 'The Nature of the Chemical Bond' (1931) and John H. Van Vleck's work on 'The Theory of Electric and Magnetic Susceptibilities' (1932). Then came the second world war, and because research gets inevitably influenced by funding, much of the efforts went in the direction of electromagnetic communications, as realized through fundamental investigations of magnetic resonance, molecular beams and laser spectroscopy.

It seems to me that it is essentially through the phenomenal studies by Walter Kohn at Carnegie

*Author for Correspondence: E-mail: sushantad@gmail.com 
Institute of Technology, now Carnegie-Mellon University of Pittsburgh and Joaquin M Luttinger at Columbia University of New York City through the 1950's that SSP began to be recognized as a subject of its own. In this context, the reader may refer to the obituary piece penned by T V Ramakrishnan at the passing of Kohn, Current Science 2016. Ramakrishnan quotes Kohn having said that when he had visited with Neils Bohr at Copenhagen in 1951, the great man had not heard the name: SSP. The subject was derisively referred to as 'Squalid State Physics'. Soon the topic got subsumed by what was dubbed 'Condensed Matter Physics' (CMP) - a sexier name, perhaps.

However, in our country, CMP began to be dominated by classical liquid-state properties, especially those that are characterized by high density (of so-called 'soft-matter') and also, the more fancy themes of 'nonlinear physics'. Another intrusion into the domain of SSP was by Statistical Mechanics. Many of us became enamoured by the formal appeal of Statistical Mechanics. The focus on Solid State Physics withered away in the maze of CMP generalities. I should clarify here that my point is not to find issue with SSP being submerged under the more expanded nomenclature of CMP; indeed, on the contrary, CMP concepts of phase transitions, complexity, emergent behaviour, nonequilibrium phenomena, etc., not only enriched SSP, but also spurred significant progress in the understanding of biological systems. What I lament is that the CMP community seemed to get (undesirably) specialized - crudely speaking - into the Planck constant $h=0$ and $h \neq 0$ sectors, in India. On the other hand, a large body of experimental data, specially emanating from our national labs, beckon attention of quantum SSP theorists.

The optimistic news is that as Solid State Physics went into a bit of a decline Nobel Prizes started to flow to areas such as High Temperature Superconductivity (Bednorz and Mueller 1987), Quantum Hall Effect (K. von Klitzing 1985, and R B Laughlin, H L Stoermer and D C Tsui 1998), Graphene (to A K Geim and K S Novoselev 2010), and more recently, to what are called topological solids (D J Thouless, J M Kosterlitz and F D M Haldane 2016). In all these systems, Quantum Mechanics is the most essential attribute.
This brings me to the lockdown period. During this time we lost a colossus of modern Solid State Physics, Philip W. Anderson (1923-2020) who ironically coined the phrase: Condensed Matter Physics and who, along with his doctoral supervisor at Harvard University, Cambridge: J H Van Vleck, earned respect for Solid State Physics by bagging the Nobel Prize in 1977. The third recipient was Nevil C Mott of Cambridge University, U K who had extensively employed Anderson's work in his own studies. Incidentally, until that time, the Nobelrecognition mostly went to particle physicists and atomic and nuclear physicists.

Anderson's research comprised beautiful and fascinating applications of quantum mechanical concepts such as to Lineshape Analysis and Pressurebroadening of Spectral lines, 'Localization' that deals with coherent destruction of the quantum phase by disorder in solids, the tricky aspect of antiferromagnetism, the Kondo effect, spin glasses, neural networks, broken symmetry ideas in superconductivity - which were a precursor to the much-celebrated 'God Particle', adaptation of Pauling's notion of resonance valence bonds to high-temperature superconducting materials, spin liquids and so on. The other remarkable facet of Anderson's work was his proximity to experimentalists - he scrupulously studied data and succeeded to make sense by intuitive and penetrative modelling with the aid of profound quantum mechanical considerations. (See the May 2020 issue of Resonance and 25 May 2020 volume of Current Science for obituary references to P. W. Anderson).

The lockdown time also witnessed the passing of one of the early protagonists of Solid State Physics in our own country: Sri Krishna Joshi (1935-2020). This leads me to analyze the state of physics in India through the independence in 1947. Prior to that the scene was dominated by spectroscopy, mainly because of the Nobel Prize-winning experiments by C V Raman on what is called the Raman Effect and his protégés such as $\mathrm{S}$ Bhagavantham and others. Came independence and major funding went to laboratories of atomic energy (DAE), space science (ISRO) and industrial research (CSIR). The forerunners of physics were individuals like Homi J Bhabha, Shanti Swarup Bhatnagar and Vikram Sarabhai - the universities went into hibernation as 
far as physics research was concerned. Entered then a man in his thirties, in a remotely-located university at Roorkee.

Joshi, until he transitioned to the CSIR system in the mid-nineteen eighties, was essentially a 'university man', having been earlier trained in Allahabad University followed by a brief sojourn to Riverside, California. At Roorkee, he created a group of young physicists who propelled emerging ideas of disordered solids such as 'spin glasses', mastered new techniques such as the coherent potential approximation and also excelled in computations of electronic structures in solids. What helped Joshi's efforts also was the generously-funded experimental activity in BARC on neutron scattering studies of lattice dynamics which hinged on phonon physics.

I conclude with where we started and that is how to resurrect quantum phenomena in solids and at the same time, rejuvenate research in universities and newly created Indian Institutes of Science Education and Research. After all, experimental work in Solid State Physics needs just modest efforts of 'small science' consisting of spectroscopic, thermodynamic and transport measurements, unlike the 'big science' of accelerators, synchrotrons, neutrino observatories, space-satellites, and so on. What is the call of the hour is to carefully sift through a large accumulation of experimental data, as was taught to us by great savants like the late Anderson. Some very pretty ideas of quantum information, memory-devices and novel material properties can emanate from such combination of efforts by experimentalists and quantum theorists. With the demise of a global harbinger of modern Solid State Physics: Anderson and its champion in India: Joshi, during this lockdown period, my thoughts invariably turned to ask the following question. Can the time be profitably utilized to think of re-invigorating research in the intertwined areas of Solid State Chemistry and Physics, to spur interdisciplinary research in materials science, in our country? That would be a real tribute to $\mathrm{P} W$ Anderson, a Foreign Fellow of the Indian National Science Academy (INSA) and S K Joshi, its past President. 